\title{
Food Safety Considerations and Research Priorities for the Cultured Meat and Seafood Industry
}

\author{
Kimberly J. Ong ${ }^{1}$, Jeremiah Johnston ${ }^{2}$, Isha Datar ${ }^{2}$, Vincent Sewalt ${ }^{3}$, Dwayne Holmes ${ }^{4}$, \\ and Jo Anne Shatkin ${ }^{1}$ \\ ${ }^{1}$ Vireo Advisors, LLC \\ ${ }^{2}$ New Harvest Inc \\ ${ }^{3} \mathrm{IFF}$ \\ ${ }^{4}$ Mosa Meat
}

September 9, 2021

\begin{abstract}
Cell-cultured meat and seafood offer a sustainable opportunity to meet the world's increasing demand for protein in a climatechanged world. A responsible, data-driven approach to assess and demonstrate safety of cell-cultured meat and seafood can support consumer acceptance and help fully realize the potential of these products. As an initial step towards a thorough demonstration of safety, this review identifies hazards that could be introduced during manufacturing, evaluates applicability of existing safety assessment approaches, and highlights research priorities that could support safe commercialization.

Input was gathered from members of the cultured meat and seafood industry, researchers, regulators, and food safety experts. A series of workshops were held with 87 industry representatives and researchers to create a modular manufacturing process diagram, which served as a framework to identify potential chemical and biological hazards along the steps of the manufacturing process that could affect the safety of a final food product. Interviews and feedback on draft documents validated the process diagram and supported hazard identification and evaluation of applicable safety methods. Most hazards are not expected to be novel; therefore, safety assessment methods from a range of fields, such as conventional and novel foods, foods produced from biotechnology, pharmaceuticals, etc., are likely to be applicable. However, additional assessment of novel inputs or products with significant differences from existing foods may be necessary. Further research on the safety of the inputs and associated residues, potential for contamination, and development of standardized safety assessment approaches (particularly animal-free methods) is recommended.
\end{abstract}

\section{Hosted file}

Ong et al Cell-cultured meat and seafood safety Review v2.pdf available at https://authorea. com/users/393371/articles/507011-food-safety-considerations-and-research-priorities-forthe-cultured-meat-and-seafood-industry 